\title{
The Effects of Gravitational Waves on the Orbital Elements of the Planets
}

\author{
M.N. Ismaiel ${ }^{1}$ and Nadia A.Saad ${ }^{2, *}$ \\ ${ }^{1}$ Astronomy Department, Faculty of Science, Al-Azhar University, Egypt \\ ${ }^{2}$ National Research Institute of Astronomy and Geophysics (NRIAG), Helwan, Egypt
}

\begin{abstract}
The present work is concerned with evaluation the effect of gravitational waves on a planetary orbit. The components of the acceleration resulting from this effect on the orbit of the planet are derived and the perturbing forces are computed. The perturbation method adopted makes use of Lagrange's planetary equations. Numerical study is done, using the Rung-Kutta fourth order method and the Mathematica software, and carried out for different sources of gravitational waves as well as the various values of planetary orbital elements.
\end{abstract}

Keywords: General Relativity, Weak Fields equations, Perturbation theories, Planetary Motion, Orbital Motion.

\section{INTRODUCTION}

In (1916) Einstien has found an error in his initial calculations which he reduced it to gravitational radiation [1]. In (1918) Einstien has corrected this error and the final results stands today as the leading -order "quadruple formula" for gravitational wave emission [2]. This formula plays a role in gravity theory analogous to the dipole formula for electromagnetic radiation, showing that gravitational waves arise from accelerated masses exactly as electromagnetic waves arise from accelerated charges. Hulse and Taylor (1975) [3] discovered and detected a pulsar as a member of a binary system. Thus for the first time it is possible to observe the gravitational interactions of a pulsar and another massive objects and determining the masses of pulsar and its companion also this discovery makes feasible a number of studies involving the physics of compact objects, the astrophysics of close binary systems, and special- and generalrelativistic effects. Nonetheless, technology has brought us to the point where detectors are now being to set interesting upper limits on gravitational waves from some sources [4]. Flanagan and Hughes [5] have illustrated the properties of gravitational waves, how it emitted and propagated in their survey about the basic of gravitational waves theory. Also, they categorized gravitational waves sources (and the method for detecting their waves) by the frequency band in which they radiate. High frequency compact sources are of stellar masses, several solar masses, while low frequency compact sources are thousand to millions of solar masses or else contain widely separated stellar mass bodies). The high frequency band $1 \mathrm{~Hz}<\mathrm{f}<10 \mathrm{~Hz}$, is targeted by the new generation of ground-based laser interferometric detectors such as LIGO (See [6]), Virgo (French-Italian detector), GEO600 [7], TAMA300 ([8] and [9]) and ACIGA [10]. The

\footnotetext{
*Address correspondence to this author at the Astronomy Department, Faculty of Science, Al-Azhar University, Egypt; Tel: 00202-22616548; Fax: 00202-22629356; E-mails: mnader_is@yahoo.com; drnadia@nriag.sci.eg,drnadiasaad@yahoo.com
}

binary star systems in which each member is a neutron star or black hole are currently the best understood sources of gravitational waves [11-14]. Stellar core collapse in massive has long been regarded as likely to be an important source of gravitational waves [15-17].

The gravitational waves in the low frequency band, $10^{-}$ ${ }^{5} \mathrm{~Hz}<\mathrm{f}<1 \mathrm{~Hz}$ is impossible to be measured using a groundbased instrument. The only way to measure these waves is to build a detector in the quit environment of space, far removed from low-frequncy noise sources. Such an instrument is currently being designed jointly by NASA in United States and ESA, the European Space Agency: LISA, the Laser Interferometer Space Antenna. If all goes well, LISA will be launched into orbit in 2018 [18].

Recently, Corda [19] had shown that the advanced projects on the detection of GWs will improve their sensitivity allowing to perform a GWs astronomy, accurate angular and frequency dependent response functions of interferometers for gravitational waves arising from various theories of gravity, i.e. general relativity and extended theories of gravity, will be the definitive test for general relativity.

It is well known that weak gravitational waves cause a change in the distance between two free particles. Several types of gravitational detectors are based on this property, including the measurement of the Doppler shift from a satellite being acted on by a gravitational wave [20]. There has been recently in the possibility of detecting gravitational waves by Doppler tracking of interplanetary spacecraft [2123]. Anderson (1971) [24] was recognized such possibilities in his inconclusive attempt to find correlations with Webber's events by examining the signal data from Mariner 6 and 7. The effects of gravitational waves on the signal have been carried out to overcome un-modeled forces such as solar wind $[20,25,26]$. The interaction of low frequency monochromatic gravitational waves with a circular Earth artificial satellite orbit has been studied by Rudenko [27]. The tidal resonance has been extended by Mashhoon [28]. Turner [29] 
used Lagrange's planetary equations to find the perturbations in the elements up to first order. He found commensurabilities for $v$ a positive integer. Nelson and Chau (1982) [30] formed the equations of variation of coordinates in an elliptic orbit and solved them numerically. They obtained analytical expressions only for normal incidence and small eccentricity, and for the $\mathrm{z}$ component of a circular orbit at oblique incidence. Ivashchenko (1987) [31] used Lagrange's planetary equations to find a first order solution in all the elements in the case of oblique incidence and found commensurabilities $v=1,2$ and 3 . Then choosing $\mathrm{I}=\pi=0$, he found the variations in $\mathrm{a}$, $\mathrm{e}$ and $\mathrm{w}$ in case of normal incidence where commensurabilities occurred for $v=1,2,3$ and 4 . In all cases he chose the time of perigee passage to zero. He expanded functions of the true anomaly in series in the mean anomaly up to order e, but when integrating neglected terms with e as a factor. Similar results were obtained by using simple averaging [32].

The present work aims to apply the methods of celestial mechanics to evaluate the effects produced by a weak gravitational wave incident on a planetary orbit. The perturbation method adopted makes use of Lagrange's planetary equations to find the perturbations in the elements. Numerical integration is done using the Rung-Kutta fourth order method, Mathematica software and is applied on the orbital motion of Venus and Pluto, which are selected taking into account their difference in the orbital elements (semi major axes $a$ and eccentricity $e$ ), with three different sources of the gravitational waves.

\section{THE EQUATION OF MOTION AND THE ACCEL- ERATION COMPONENTS}

General relativity predicts gravitational waves as freely propagating "ripples" in space time. Far away from the source one can use the weak field approximation to express the curvature tensor $g_{\mu \nu}$ as a small perturbation $h_{\mu \nu}$ of the Minkowski metric $\eta_{\mu v}[27,33]$

$$
g_{\mu v}=\eta_{\mu v}+h_{\mu v}
$$

with $h_{\mu v} \prec \prec 1$.

Using this assumption to solve the Einstien field equations in vacuum yields a normal wave equation. Using the transverse traceless gauge its general solutions can be written as

$$
h_{\mu v}=h_{\leftarrow}\left(\frac{t-z}{c}\right)+h_{\times}\left(\frac{t-z}{c}\right)
$$

where $\mathrm{z}$ is the direction of propagation, $h_{\leftarrow}$ and $h_{\times}$are the two polarizations, then

$$
h_{+}\left(\frac{t-z}{c}\right)+h_{\times}\left(\frac{t-z}{c}\right)=\left[\begin{array}{cccc}
0 & 0 & 0 & 0 \\
0 & h_{+} & h_{\times} & 0 \\
0 & -h_{\times} & h_{+} & 0 \\
0 & 0 & 0 & 0
\end{array}\right] e^{i \omega t-i k z}
$$

The Lorentz gauge condition implies that the spatial metric perturbation is transverse i.e $\partial_{z} h_{i k}=0$, this yields $h_{i k}(t-z)=$ constant. This constant must be zero to satisfy the condition that $h_{a b} \rightarrow 0$ as $r \rightarrow \infty$. The only non-zero components of $h_{i j}$ are then $\mathrm{h}_{\mathrm{xx}}, \mathrm{h}_{\mathrm{xy}}, \mathrm{h}_{\mathrm{yx}}$, and $\mathrm{h}_{\mathrm{yy}}$. Symmetry and the trace free condition further mandate that only two of these are independent

$$
\begin{aligned}
& h_{x x}=-h_{y y}=h_{+}(t-z) \\
& h_{x y}=-h_{y x}=h_{\times}(t-z)
\end{aligned}
$$

So that the separation between two particles could be determined by

$$
d \ell^{2}=d z^{2}+\left(1-h_{x x}\right) d x^{2}+\left(1+h_{x x}\right) d y^{2}+2 h_{x y} d x d y
$$

Under such conditions the wave creates a field of variable accelerations with the components $[34,35]$ :

$$
f_{x}=h_{1} x+h_{2} y, f_{y}=h_{2} x-h_{1} y, f_{z}=0
$$

where $h_{1}=\frac{1}{2} \frac{\partial^{2} h_{x x}}{\partial t^{2}}, h_{2}=\frac{1}{2} \frac{\partial^{2} h_{x y}}{\partial t^{2}}$

and $f_{x}, f_{y}$, and $f_{z}$ are the components of the acceleration vector at the point with coordinates $(\mathrm{x}, \mathrm{y}, \mathrm{z})$,

and $h_{x x}=h_{+} \cos \left(n_{g} t+\alpha_{1}\right), h_{x y}=h_{\times} \cos \left(n_{g} t+\alpha_{2}\right)$

Therefore we can write $h_{1}$ and $h_{2}$ in the form

$h_{1}=\beta \cos \left(n_{g} t+\alpha_{1}\right)$ and $h_{2}=\gamma \cos \left(n_{g} t+\alpha_{2}\right)$

where $\beta=-\frac{1}{2} h_{+} n_{g}^{2} \quad, \gamma=-\frac{1}{2} h_{\times} n_{g}^{2}$, and $n_{g}, h_{+}$, and $h$ are respectively the frequency and the dimensionless amplitudes of the wave in two orthogonal directions in the transverse plane.

Regarding the estimates of the frequencies and amplitudes of gravitational waves from different sources, we can fairly assume that $\beta$ and $\gamma$ are of the order of planetary eccentricity.

\section{THE DISTURBING FUNCTION}

The effects of the gravitational wave, on the two masses system $m_{1}$ and $m_{2}$ moving along elliptical orbit under the action of mutual gravity and the incident gravitational wave, causes the appearance of an additional weak perturbing force with the components represented by equation (6). From this components of acceleration vector we find a potential like function $\mathrm{V}$ such that

$$
\bar{r}=-\frac{\partial V}{\partial r}=\frac{\partial R}{\partial r}
$$

and the disturbing function $\mathrm{R}$, will be in the form 


$$
R=-\frac{1}{2} h_{1}\left(y^{2}-x^{2}\right)+h_{2} x y
$$

We can express $\mathrm{R}$ in terms of the orbital elements, using the expressions

$$
\begin{gathered}
x=r(\cos \Omega \cos (f+w)-c \sin \Omega \sin (f+w)) \\
y=r(\sin \Omega \cos (f+w)+c \cos \Omega \sin (f+w))
\end{gathered}
$$

Then

$$
\begin{aligned}
& \frac{d \Omega}{d t}=\frac{1}{n a^{2} \sqrt{1-e^{2}} \sin i} \frac{\partial R}{\partial i} \\
& \frac{d \omega}{d t}=-\frac{\cos i}{n a^{2} \sqrt{1-e^{2}} \sin i} \frac{\partial R}{\partial i}+\frac{\sqrt{1-e^{2}}}{n a^{2} e} \frac{\partial R}{\partial e}
\end{aligned}
$$

Where $\mathrm{n}$, denote the satellite's mean motion, and $a, e, i$, $\Omega, \omega, M$ are the Keplerian orbital elements.

$$
\begin{aligned}
R= & -\frac{1}{16} \varepsilon^{2}\left\{2 s ^ { 2 } \left[\operatorname{coc}\left(2 \Omega+n_{g} t+\alpha_{1}\right)+\operatorname{coc}\left(2 \Omega-n_{g} t-\alpha_{1}\right)\right.\right. \\
& \left.\quad-A\left(\sin \left(2 \Omega+n_{g} t+\alpha_{2}\right)+\sin \left(2 \Omega-n_{g} t-\alpha_{2}\right)\right)\right] \\
+ & \left(c^{2}+2 c+1\right)\left[\operatorname{coc}\left(2 f+2 w+2 \Omega+n_{g} t+\alpha_{1}\right)+\operatorname{coc}\left(2 f+2 w+2 \Omega-n_{g} t-\alpha_{1}\right)\right. \\
& \quad-A\left(\sin \left(2 f+2 w+2 \Omega+n_{g} t+\alpha_{2}\right)+\sin \left(2 f+2 w+2 \Omega-n_{g} t-\alpha_{2}\right)\right. \\
+ & \left.\left.\sin \left(2 f+2 w-2 \Omega+n_{g} t+\alpha_{2}\right)+\sin \left(2 f+2 w-2 \Omega-n_{g} t-\alpha_{2}\right)\right)\right]+ \\
+ & \left.\left(c^{2}-2 c+1\right)\left[\operatorname{coc}\left(2 f+2 w-2 \Omega+n_{g} t+\alpha_{1}\right)+\operatorname{coc}\left(2 f+2 w-2 \Omega-n_{g} t-\alpha_{1}\right)\right]\right\}
\end{aligned}
$$

where $\varepsilon$ is a small parameter defined as $\varepsilon=-\beta=\frac{1}{2} n_{g}^{2} h_{+}, \quad A=-\frac{h_{\times}}{h_{+}}, c=\cos i$

and $s=\sin i$.

To develop the expression of $\mathrm{R}$ in terms of the mean anomaly $\mathrm{M}$, the terms

$\left(\frac{r}{a}\right)^{n} \cos m f$ and $\left(\frac{r}{a}\right)^{n} \sin m f$ are expanded as a function of the mean anomaly [36]. Then after some little reductions $\mathrm{R}$ is obtained.

\section{THE EQUATIONS FOR THE VARIATION OF THE ELEMENTS}

To . find the variation of the orbital elements $a, e, I, \Omega, \omega$ and $M$, due to the effects of the gravitational waves on the motion of a planet, we shall use the Lagrange's planetary equations [36, 37] and developing the equations of motion with the mean anomaly as the independent variable

$\frac{d a}{d t}=\frac{2}{n a} \frac{\partial R}{\partial M}$

$\frac{d e}{d t}=\frac{1-e^{2}}{n a^{2} e} \frac{\partial R}{\partial M}-\frac{\sqrt{1-e^{2}}}{n a^{2} e} \frac{\partial R}{\partial \omega}$

$\frac{d i}{d t}=\frac{\cos i}{n a^{2} \sqrt{1-e^{2}} \sin i} \frac{\partial R}{\partial \omega}-\frac{1}{n a^{2} \sqrt{1-e^{2}} \sin i} \frac{\partial R}{\partial \Omega}$

\section{THE RESULTS}

We have constructed a computer program using a Mathematica package to calculate the perturbing force and the disturbing function $R$, due to the effect of the gravitational wave, and substituting into the Lagrange equations for the Keplarian set of elements equation (14). We have programmed all these Equations of motion and applied it on the orbital motion of Venus and Pluto, which are selected taking into account their difference in the orbital elements (semi major axes $a$ and eccentricity $e$ ), with three different sources of the gravitational waves. Details of the characteristics of these waves are shown in Table I [38] and the gravitational wave amplitudes are taken from [23]. The mean orbital elements corresponding to certain epoch and other planets details are provided in Table II.

Our results are shown in Table III, which provides the variation in the orbital parameters $a, e, I, \omega, \Omega$ and the perturbed acceleration, obtained by direct numerical integration of the equations of motion.

\section{CONCLUSION}

Numerical studies are done with three different sources of gravitational wave differ in their properties which selected to cover a wide range of frequency. Numerical comparison between the effects of these waves on two systems of masses (Sun-Venus) and (Sun-Pluto) are made. The comparison shows that the orbit is deformed with changes in the semimajor axis and eccentricity. The results illustrate, that the effects of gravitational waves are too small, but are not completely negligible and the effects of gravitational waves on the closed bounded system (Sun-Venus) are smaller than the faraway bounded one (Sun-Pluto). That means the effects of the gravitational waves are weak on the orbital elements of bodies with small separation distance and the effect increase 
Table I. Characteristics of the Gravitational Wave from Different Sources

\begin{tabular}{|c|c|c|c|}
\hline Source & Frequency (Hz) & Wave Length & Amplitude \\
\hline \hline Primordial & $10^{-16}$ & $\sim 10^{9}$ lt.yrs & $3-210 \times 10^{-24}$ \\
\hline Primordial cosmic strings & $10^{-9}$ & $\sim 10$ lt.yrs & $0.01-2 \times 10^{-22}$ \\
\hline $\begin{array}{c}\text { Binary stars, Super-massive black } \\
\text { holes }\end{array}$ & $10^{-4}$ to $10^{-1}$ & $\sim 0.01 \mathrm{Au}$ \\
to $10 \mathrm{Au}$ & $\sim 300 \mathrm{~km}$ \\
\hline $\begin{array}{c}\text { Inspirals:NS+NS,BH+BH,NS+BH } \\
\text { to } 30000 \mathrm{~km}\end{array}$ & $0.02-1 \times 10^{-20}$ \\
\hline Supernovae,Spinning neutron stars & 10 to $10^{3}$ & $\sim 300 \mathrm{~km}$ & $0.001-001 \times 10^{-18}$ \\
\hline
\end{tabular}

Table II. The Orbital Elements of Venus and Pluto Reference Date: 12:00 UT 1 Jan 2000

\begin{tabular}{|c|c|c|}
\hline Parameter & Venus & 39.49245 \\
\hline \hline Semi major axis (AU) & 0.723643504 & 0.249 \\
\hline Eccentricity & 0.013 & 17.1 \\
\hline Inclination (Deg.) & 3.39 & 110.3 \\
\hline R.A. of ascending Node (Deg) & 76.6 & 114.0 \\
\hline Argument of perigee (Deg) & 132 & 0.003973966 \\
\hline Mean Motion (Deg/day) & 1.60216874 & 1990 Feb 5 \\
\hline Time of Periapsis(UT) & 1998 Mar 2 & 248.0208 years \\
\hline Orbital Period & $224.695434($ days) & $0.1783 R_{E}$ \\
\hline Equatorial Radius & $0.9488498 R_{E}$ & \\
\hline
\end{tabular}

Table III. The Variation of the Orbital Elements and the Total Perturbed Acceleration

\begin{tabular}{|c|c|c|c|c|c|c|c|}
\hline \multicolumn{2}{|c|}{ Parameter } & \multirow{2}{*}{$\frac{\mathbf{a}(\mathbf{A U}) * \mathbf{1 0}}{1.305583 \times 10^{-16}}$} & \multirow{2}{*}{$\frac{\mathbf{e}}{6.79491 \times 10^{-15}}$} & \multirow{2}{*}{$\begin{array}{c}\mathbf{I} \\
8.88178 \times 10^{-15}\end{array}$} & \multirow{2}{*}{$\frac{\omega}{6.245 \times 10^{-17}}$} & \multirow{2}{*}{$\begin{array}{c}\boldsymbol{\Omega} \\
0\end{array}$} & \multirow{2}{*}{$\begin{array}{c}\mathbf{f} \\
2.9613407 \times 10^{-18}\end{array}$} \\
\hline V & Sour1 & & & & & & \\
\hline s & Sour3 & 0 & $5.20417 \times 10^{-18}$ & 0 & 0 & 0 & $1.01705 \times 10^{-21}$ \\
\hline \multirow{2}{*}{$\begin{array}{l}\mathrm{P} \\
1 \\
\mathrm{u} \\
\mathrm{t} \\
\mathrm{o}\end{array}$} & Sour2 & $8.43067 \times 10^{-5}$ & $4.02085 \times 10^{-6}$ & $6.37779 \times 10^{-12}$ & $9.92906 \times 10^{-12}$ & $1.48725 \times 10^{-12}$ & $1.87147 \times 10^{-15}$ \\
\hline & Sour3 & $5.00592 \times 10^{-10}$ & $2.38724 \times 10^{-11}$ & $1.33227 \times 10^{-15}$ & $2.05391 \times 10^{-15}$ & 0 & $4.03417 \times 10^{-20}$ \\
\hline
\end{tabular}

when the separated distance between the two masses increase.

\section{REFERENCES}

[1] Einstien A. Approximative integration of the field equations of gravitation. Sitzungsberichte PreuBische Akad der Wiss Berlin Math Phys 1916; 688.

[2] Einstein A. On gravitational waves. Sitzungsberichte PreuBische Akad der Wiss Berlin Math Phys 1918; 154.

[3] Hulse RA, Taylor JH. Discovery of a pulsar in a binary system. Astrophys J Lett 1975; 195: L51-3.
[4] Abbott B, Abbott R, Adhikari R et al. First upper limits from LIGO on gravitational wave bursts. Phys Rev D 2004; 69:102001.

[5] Flanagan E, Hughes S. The basic of gravitational wave theory. New J Phys 2003; 7: 204.

[6] See www.ligo.caltech.edu.

[7] Luck H, Aufmuth, P, Brozek OS, et al. Proceeding of the 3rd Edoardo Amaldi Conference. Meshkov S, Ed, AIP Conference Proceedings 523, Melville, New York 2000: p.119.

[8] Ando M, Arai K, Takahashi R, et al. Stable operation of a 300-m laser interferometer with sufficient sensitivity to detect gravitational wave events within our galaxy. Phys Rev Lett 2001; 86: 3950. 
[9] Kuroda K, Ohashi M, Miyoki S, et al. Large scale cryogenic gravitational wave telescope. Int J Mod Phys D 1999; 8: 557.

[10] McCleland D, Gray MB, Shaddock DA, et al. Proceeding of 3rd Edoardo Amaldi Conference. Meshkov S, Ed. AIP Conference Proceedings 523. New York: Melvill 2000, p. 140.

[11] Narayan R, Piran T, Shemi A. Neutron star and black hole binaries in the Galaxy. Astrophys J 1991; L17: 379.

[12] Phinney ES. The rate of neutron star binary mergers in the universe-Minimal predictions for gravitational wave detectors. Astrophys J 1991; L17: 380.

[13] Kalogera V, Lorimer DR. An upper limit on the coalescence rate of double neutron star binaries in the galaxy. Astrophys J 2000; 530: 890.

[14] Burgay M, D'Amico N, Possenti A, et al. An increased estimate of the merger rate of double neutron stars from observations of a highly relativistic system. Nature 2003; 426: 531 .

[15] Eardley DM. Gravitational Radiation, In: Dereulle N, Piran T, Eds, Amsterdam: North Holland 1983: p. 257.

[16] Chandrasekhar S. Ellipsoidal figures of equilibrium. New Haven: Yale University Press 1969.

[17] Fryer CL, Holz DE, Hughes SA. Gravitational wave emission from core collapse of massive stars. Astrophys J 2002; 565: 430.

[18] http://www.lisa.aei-hannover.de

[19] Corda C. Interferometric detection of gravitational waves: the definitive test for General Relativity. Int J Modern Phys D 2009; D18: 2275-82

[20] Estabrook FB, Wahlquist HD. Response of Doppler spacecraft tracking to gravitational radiation. Gen Relat Gravitation 1975; 6: 439-47.

[21] Davies RW. Ondes et Radiations Gravitationelles, ed. Y.ChoquetBruhat, Colloq Intern CNRS, No 2201974.

[22] Anderson AJ. Experimental Gravitation, In: Bertotti B, Eds. New York: Academic Press 1974: p. 163.
[23] Douglass DH, Braginsky VB. Einstein Centenary Volume, In: Hawking S, Isreal W, Eds. Cambridge: Cambridge University Press 1979.

[24] Anderson AJ. Probability of long period gravitational radiation. Nature 1971; 229: 547.

[25] Kundt W. Dragfree spaceprope. Astrophys Space Sci 1974; 30: 455.

[26] Wahlquist HD, Anderson JD, Estabrook FB, Thorne KS. 1976, Proc Intern Symposium on Experimental Gravitation, Rome. A ccademia Nazionale die Lincei 1971.

[27] Rudenko VN. Test bodies under the effect of gravitational radiation. Sov Astron 1975; 19: 270.

[28] Mashhoon B. On the detection of gravitational radiation by the Doppler tracking of spacecraft. Astrophys J 1979; 227: 1019.

[29] Turner MS. Influence of a weak gravitational wave on a bound system of two point-masses. Astrophys J 1979; 233: 685.

[30] Nelson LA, Chau WY. Orbital perturbations of a gravitationally bound two-body system with the passage of gravitational waves. Astrophys J 1982; 254: 735.

[31] Ivanshchenko AV. Variation of the Keplerian elements of a planetary orbit under the action of a gravitational wave. Sov Astron 1987; 31: 76.

[32] Kochkin RA, Shytov YG. Influence of a Periodic Gravitational Wave on the Parameters of a Binary System. Sov Astron 1987; 31: 537.

[33] Futamase T, Matsuda T. Resonance between Primordial Gravitational Waves and Gravitationally Bound Systems. Prog Theore Fiz 1979; 61: 86

[34] Ivanshchenko, AV. Dep VINITI 1975; No 1713: 75.

[35] Ivanshchenko AV. Izv Vyssh Uchebn Zaved Fiz 1983; No 4:128.

[36] Smart WM. Celestial Mechanics. New York John Wiley 1961.

[37] Brouwer D, Clemence GM. Methods of Celestial Mechanics. New York: London Academic Press 1961.

[38] Sigg D. Gravitational Wave Sources and Detection; LIGOP9800007-00-D 1998;

(c) Ismaiel and Saad; Licensee Bentham Open.

This is an open access article licensed under the terms of the Creative Commons Attribution Non-Commercial License (http://creativecommons.org/licenses/by-nc/3.0/) which permits unrestricted, non-commercial use, distribution and reproduction in any medium, provided the work is properly cited. 